\title{
A Generalizable Hierarchical Bayesian Model for Persistent SAR Change Detection
}

\author{
Gregory E. Newstadt ${ }^{a}$, Edmund G. Zelnio ${ }^{b}$, and Alfred O. Hero III ${ }^{a}$ \\ ${ }^{a}$ Electrical Engineering and Computer Science, University of Michigan, Ann Arbor, MI \\ 48109, USA; \\ ${ }^{b}$ Air Force Research Laboratory, Wright Patterson Air Force Base, OH, 45433, USA
}

\begin{abstract}
This paper proposes a hierarchical Bayesian model for multiple-pass, multiple antenna synthetic aperture radar (SAR) systems with the goal of adaptive change detection. We model the SAR phenomenology directly, including antenna and spatial dependencies, speckle and specular noise, and stationary clutter. We extend previous work ${ }^{1}$ by estimating the antenna covariance matrix directly, leading to improved performance in high clutter regions. The proposed SAR model is also shown to be easily generalizable when additional prior information is available, such as locations of roads/intersections or smoothness priors on the target motion. The performance of our posterior inference algorithm is analyzed over a large set of measured SAR imagery. It is shown that the proposed algorithm provides competitive or better results to common change detection algorithms with additional benefits such as few tuning parameters and a characterization of the posterior distribution.
\end{abstract}

Keywords: synthetic aperture radar, change detection, hierarchical Bayesian model.

\section{INTRODUCTION}

SAR radars traditionally are used to image stationary objects by integrating radar returns from spatially diverse points. SAR images are often formed with much longer integration times than other radar modes (in particular, MTI-mode radars). While this leads to high resolution images in many cases, the long integration times also lead to other undesirable effects. Moving objects can cause phase errors in the reconstruction of SAR images, leading to well known dispersion and displacement effects. ${ }^{2}, 3$ Moreover, angular scintillation (aka 'specular noise' or 'glints') can cause large phase errors that may considerably degrade signal quality. ${ }^{4}$ As the name suggests, angular scintillation has a large angular dependence in the sense that the intensity of this noise source is only large from few azimuth angles. Speckle noise is an additional noise source that arises from coherent imaging with SAR. Speckle noise tends to be spatially correlated, depending on the texture of the surrounding pixels (e.g., buildings versus vegetation.) Unlike specular noise, the intensity of speckle noise tends to be uniform as a function of azimuth angle.

In this work, we focus on the situation where the scene is being persistently monitored. Thus, images are available from multiple passes of the radar platform, multiple antennas (phase centers), and multiple lookangles. However, the background of these images remains relatively unchanged and is thus modeled as being embedded in a low-dimensional subspace. Exploiting the low-dimensional subspace in order to extract the moving targets of interest is used by many common algorithms. Soumekh ${ }^{5}$ shows that all stationary objects can be removed from an ideal set of SAR images formed in a monopulse radar system by using a simple difference image, also known as displaced phase center array (DPCA) processing. In practice, the antennas are not perfectly calibrated, which can significantly degrade the performance of DPCA. Signal subspace processing $^{6}$ (SSP) addresses this problem by proposing an adaptive blind calibration technique of a two

Further author information: (Send correspondence to Gregory E. Newstadt at newstage@umich.edu)

This document was cleared for public release under document number 88 ABW-12-2639. 
channel system that assumes that the signals are related through a locally spatially invariant filter. Ender also considers using multiple channels for SAR detection of moving targets through space-time adaptive processing $^{7}$ (STAP), which adaptively estimates the clutter covariance matrix in order to separate moving targets from the background. However, STAP is limited in the sense that it requires target-free data in order to effectively estimate the clutter covariance matrix. In our work, we estimate both the calibration constants and the clutter covariance directly in order to extract the moving targets from the SAR images.

Previously, we presented a model for synthetic aperture radar (SAR) imagery in order to separate a sparse foreground component that may contain targets of interest from the stationary background. ${ }^{1}$ A hierarchical Bayesian formulation was proposed that extends the model of Ding, He and Carin ${ }^{8}$ using the methods of Tipping $^{9}$ and Wipf and Rao. ${ }^{10,11}$ This model

1. Provided Monte Carlo estimates of the posterior distribution using an efficient MCMC sampler.

2. Used conjugate distributions with non-informative hyperparameters, leading to very few tuning parameters.

3. Had low reconstruction errors and robustness to noise parameters on a synthetic dataset.

4. Performed well on a limited set of measured SAR imagery.

In this paper, we extend this model in several ways that include

1. Estimating the antenna covariance matrix directly, leading to improved performance in both low and high variance regions of the scene.

2. Generalizing the model to be able to account for prior knowledge on the locations of the target signature.

3. Generalizing the model to include spatial dependencies using a hidden Markov model.

4. Analyzing the performance of our algorithm over a large set of measured SAR imagery with

- Comparisons to common change detection algorithms such as DPCA and along-track interferometry (ATI).

- Comparisons as a function of our model (i.e., with/without prior knowledge and/or an antenna covariance model).

- Analysis as a function of integration length and the number of observations.

The rest of the paper is organized as follows. The SAR image model is given in Section 2 . Section 3 discusses the Monte Carlo implementation for posterior inference on the model. Performance of the model and inference is analyzed with both synthetic and measured SAR imagery in Section 4. Finally, conclusions and future work are provided in Section 5.

\section{SAR IMAGE MODEL}

In this work, it is assumed that we have access to SAR images formed from multiple passes of a radar platform with multiple antennas (i.e., phase centers.) Moreover, images are formed over distinct azimuth angle ranges that can be indexed by the frame number, $f$. Table 1 provides the indexing scheme used throughout this paper in order to distinguish between images from various antennas, frames, and/or passes.

The image model proposed in this paper is very similar to our previous work. ${ }^{1}$ Whenever relevant, we will point out the differences in our models. 
Table 1. Index variable descriptions

\begin{tabular}{|l|c|c|}
\hline \multicolumn{1}{|c|}{ Index Description } & Index Variable & Range \\
\hline Antenna (channel) & $k$ & $1,2, \ldots, K$ \\
\hline Frame (azimuth range) & $f$ & $1,2, \ldots, F$ \\
\hline Pass & $i$ & $1,2, \ldots, N$ \\
\hline Pixel & $p$ & $1,2, \ldots, P$ \\
\hline
\end{tabular}

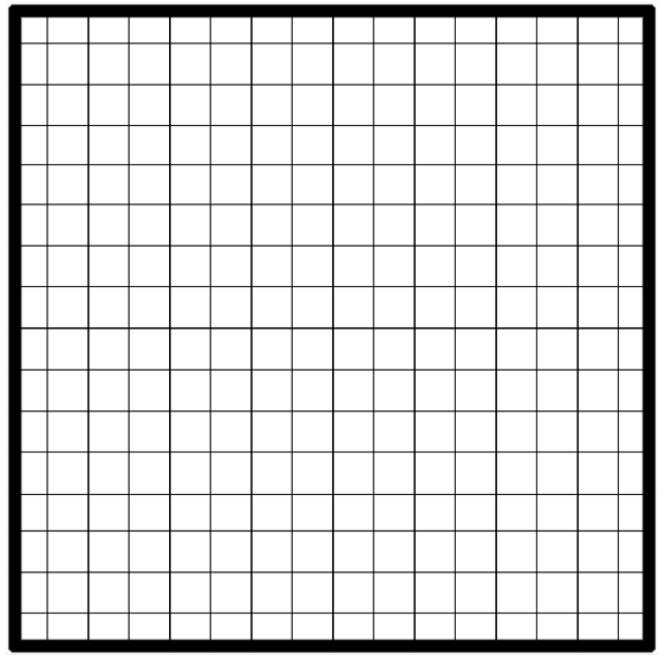

(a) Independent pixels

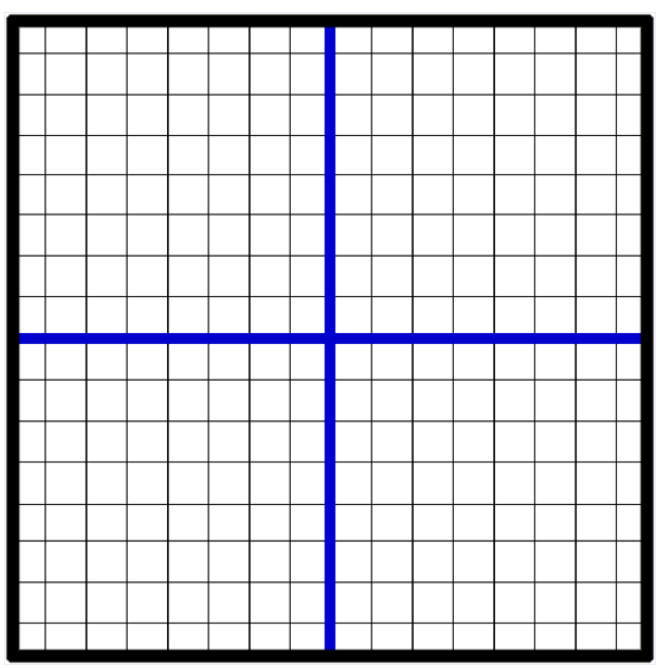

(c) Locally identical distributions (large)

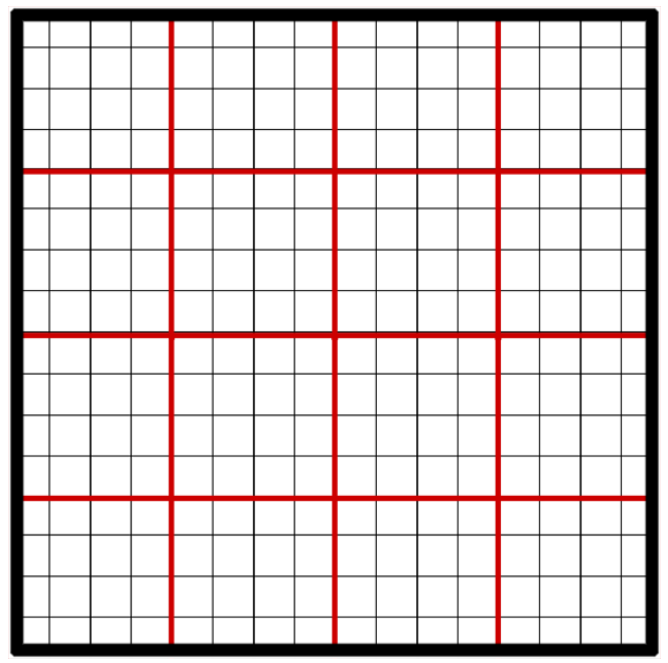

(b) Locally identical distributions (medium)

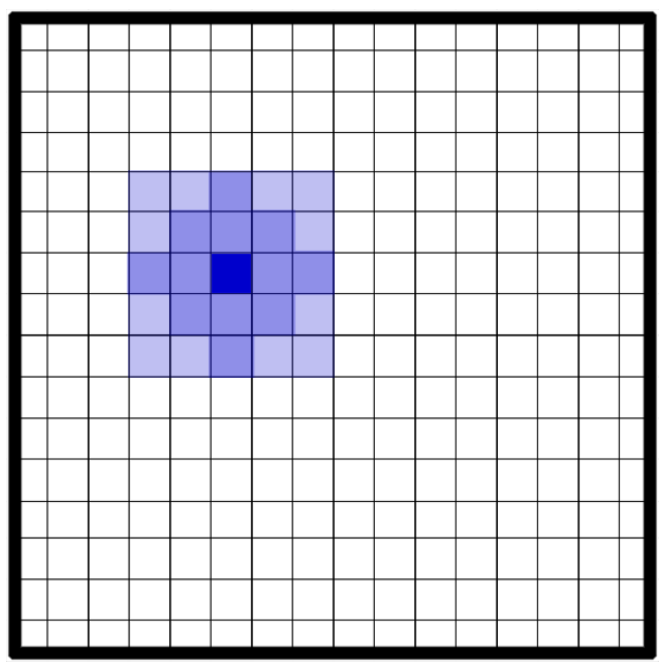

(d) HMM dependence for a single pixel

Figure 1. Various scenarios for spatial dependence used in this paper. In (a), pixels are independent. In (b) and (c), pixels share an identical distribution over a medium and large area, respectively. In (d), pixels are assumed to have a hidden Markov model dependence on neighboring cells with higher probabilities close to the center pixel. 
Similar to our previous work, we propose a decomposition of SAR images into a background component that lies in a low-dimensional subspace, a foreground component that is sparse, and additive noise:

$$
I_{k, f, i}(p)=H_{k, f, i}(p)\left(B_{k, f, i}(p)+T_{k, f, i}(p)\right)+V_{k, f, i}(p),
$$

where $H_{k, f, i}(p)$ is a spatially varying filter, $B_{k, f, i}(p)$ is the background component, $T_{k, f, i}(p)$ is the foreground component, and $V_{k, f, i}(p)$ is zero-mean additive noise. Moreover, we decompose the background component as

$$
B_{k, f, i}(p)=S_{k, f}(p)+X_{k, f, i}(p)+\delta_{f}(p) Y_{k, f, i}(p),
$$

where $S_{k, f}(p)$ is the inherent background that is identical over all passes, $X_{k, f, i}(p)$ is the speckle noise component, $Y_{k, f, i}(p)$ is the specular noise (glint) component, and $\delta_{f}(p)$ is an indicator variable for specular noise. The foreground component decomposes as

$$
T_{k, f, i}(p)=D_{f, i}(p) M_{k, f, i}(p),
$$

where $M_{k, f, i}(p)$ is the target value and $D_{f, i}(p)$ is an indicator variable for the existence of the target at that pixel. Table 2 provides the distributional models of each of these variables. The remainder of the section discusses the model in greater detail.

Table 2. Distributional models for each component in equations (1), (2), and (3)

\begin{tabular}{|l|ccccc|c|}
\hline \multicolumn{1}{c}{ Component } & Variable & Distribution & Parameters & Region & All $k ?$ \\
\hline Calibration constant & $H_{k, f, i}(p)=h_{k, f, i}(g)$ & Deterministic $(\mathrm{LS})$ & none & $p \in Z_{g}$ & No \\
\hline Stationary & $S_{k, f}(p)$ & Normal $\left(0, \boldsymbol{\Sigma}^{S}(j)\right)$ & $\boldsymbol{\Sigma}^{S}(j)$ & $p \in Q_{j}$ & Yes \\
\hline Speckle & $X_{k, f, i}(p)$ & Normal $\left(0, \boldsymbol{\Sigma}^{X}(j)\right)$ & $\boldsymbol{\Sigma}^{X}(j)$ & $p \in Q_{j}$ & Yes \\
\hline Specular (glint) & $Y_{k, f, i}(p)$ & Normal $\left(0, \boldsymbol{\Sigma}^{Y}(j)\right)$ & $\boldsymbol{\Sigma}^{Y}(j)$ & $p \in Q_{j}$ & Yes \\
\hline Specular indicator & $\delta_{f}(p)$ & Bernoulli $\left(\pi_{f}^{Y}(p)\right)$ & $\pi_{f}^{Y}(p)$ & Each $p$ & No \\
\hline Target & $M_{k, f, i}(p)$ & Normal $\left(0, \boldsymbol{\Sigma}^{M}\right)$ & $\boldsymbol{\Sigma}^{M}$ & All $p$ & Yes \\
\hline Target indicator & $D_{f, i}(p)$ & Bernoulli $\left(\pi_{f, i}^{M}(p)\right)$ & $\pi_{f, i}^{M}(p)$ & Each $p$ & No \\
\hline Additive noise & $V_{k, f, i}(p)$ & Normal $\left(0, \boldsymbol{\Sigma}^{V}\right)$ & $\boldsymbol{\Sigma}^{V}$ & All $p$ & Yes \\
\hline Class assignment & $\vec{d}(p)$ & Multinomial $(1 ; \vec{q})$ & $\vec{q}$ & Each $p$ & No \\
\hline
\end{tabular}

\subsection{Calibration constants, $H_{k, f, i}(p)$}

The calibration constants are assumed to be constant within small spatial regions as denoted by either Figure 1(b) or 1(c). The calibration constant within such a region is a single value $h_{k, f, i}(g)$ for all pixels $p \in Z_{g}$. In general, $h_{k, f, i}(g)$ may be randomly distributed, though experimental evidence suggests that using least-squares is sufficient for estimating the values.

\subsection{Antenna covariance models for $\Sigma$}

Each pixel in the stationary, speckle, specular, and target value components is jointly distributed across the $K$ antennas. Since the pixel values are complex, the joint distribution is over $2 K$ values. Consider a zero-mean vector $W=\left[\begin{array}{llll}w_{1} & w_{2} & \cdots & w_{K}\end{array}\right]^{T}$, where

$$
w_{k}=a_{k}+j b_{k}, \quad k=1,2, \ldots, K .
$$


In this work, $W$ could be representative of $\left\{S_{k, f}(p)\right\}_{k},\left\{X_{k, f, i}(p)\right\}_{k},\left\{Y_{k, f, i}(p)\right\}_{k}$ or $\left\{M_{k, f, i}(p)\right\}_{k}$. The complex covariance matrix is given by

$$
\boldsymbol{\Gamma}=E\left[W W^{H}\right] \in \mathbb{C}^{K \times K},
$$

which can be related to the real-valued covariance matrix

$$
\boldsymbol{\Sigma}=\frac{1}{2}\left[\begin{array}{cc}
\operatorname{Re}\{\boldsymbol{\Gamma}\} & -\operatorname{Im}\{\boldsymbol{\Gamma}\} \\
\operatorname{Im}\{\boldsymbol{\Gamma}\} & \operatorname{Re}\{\boldsymbol{\Gamma}\}
\end{array}\right] \in \mathbb{R}^{2 K \times 2 K} .
$$

In this work, we assume a specific structure for the complex covariance matrices $\boldsymbol{\Gamma}$ which can be derived by assuming that

$$
E\left[\left(w_{k}\right)\left(w_{j}\right)^{*}\right]=\left\{\begin{array}{ll}
\sigma^{2}, & k=j \\
\sigma^{2} \rho e^{-j \phi_{k j},} & k \neq j
\end{array} \quad k, j=1,2, \ldots, K\right.
$$

and

$$
E\left[\left[\begin{array}{l}
a_{k} \\
b_{k}
\end{array}\right] \mid\left[\begin{array}{l}
a_{j} \\
b_{j}
\end{array}\right]\right]=\boldsymbol{P}_{\phi_{j k}}\left[\begin{array}{c}
a_{j} \\
b_{j}
\end{array}\right] \quad k, j=1,2, \ldots, K
$$

where $\boldsymbol{P}_{\phi}$ is a rotation matrix by angle $\phi$. In particular, let

$$
\boldsymbol{\Gamma}=\sigma^{2}\left[\begin{array}{cccc}
1 & \rho e^{-j \phi_{12}} & \cdots & \rho e^{-j \phi_{1 K}} \\
\rho e^{-j \phi_{21}} & 1 & \cdots & \rho e^{-j \phi_{2 K}} \\
\vdots & \vdots & \ddots & \vdots \\
\rho e^{-j \phi_{K 1}} & \rho e^{-j \phi_{K 2}} & \cdots & \rho e^{-j \phi_{K K}}
\end{array}\right]
$$

where $\sigma^{2}$ is the channel variance, $\rho$ is the coherence between antennas, and $\left\{\phi_{n m}\right\}_{n, m}$ are the phase differences between the channels. Note that for background components, $\rho$ should be near one and $\phi_{i j} \approx 0$. A more general model could account for different channel variance and coherence values, but since we use the calibration constants $H_{k, f, i}(p)$ to equalize the channels, the effect was seen to be relatively insignificant.

It should be noted that this form of the covariance matrix is related directly to some of the very common methods for change detection in SAR imagery. In particular, consider the two antenna case $(K=2)$. It can easily be shown that the eigendecomposition of $\boldsymbol{\Sigma}$ leads to eigenvalues $\lambda$ and eigenvectors $\nu$ :

$$
\begin{aligned}
& \lambda(\boldsymbol{\Sigma})=\left\{2 \sigma^{2}(1+\rho), 2 \sigma^{2}(1-\rho)\right\} \\
& \nu(\boldsymbol{\Sigma})=\left\{\left[\begin{array}{c}
1 \\
e^{-j \phi}
\end{array}\right],\left[\begin{array}{c}
1 \\
-e^{-j \phi}
\end{array}\right]\right\} .
\end{aligned}
$$

When the phase $\phi$ is zero, the second eigenvector reduces to $[1-1]^{T}$, which can be interpreted as DPCA for a two-antenna system. Moreover, along-track interferometry (ATI), which thresholds the phase $\phi$, clearly depends on the eigenvalues in a direct manner. Deming ${ }^{12}$ shows that ATI performs well when canceling bright clutter (i.e., high $\sigma^{2}$ and $\rho \approx 1$ ), while DPCA performs well for canceling dim clutter (i.e., small $\sigma^{2}$ and $\rho \approx 0$.) In our work, we hope to gain the discriminating power of both DPCA and ATI by modeling the covariance matrices directly.

Furthermore it should be noted that this covariance model generalizes our previous model ${ }^{1}$ which set $\rho=1$ and $\phi_{n m}=0$ for all $n \neq m$ for the stationary, speckle and specular components. In Section 4 , we compare the performance of both models with measured SAR imagery. 


\subsection{Spatial dependencies for the background components}

In our previous model, ${ }^{1}$ we assumed that objects in the background can be defined by one of $C$ classes (e.g., roads, vegetation, or buildings.) Let

$$
\vec{d}(p)=\left\{d_{c}\right\}_{c=1}^{C} \sim \operatorname{Multinomial}\left(1 ; q_{1}, q_{2}, \ldots, q_{C}\right)
$$

where $q_{n}$ is the prior probability of the $c$-th region type. Then the class assignment is the single location in $\vec{d}$ with value equal to one. Since object classes tend to have high spatial dependencies, the previous model assumed that pixels within a small neighborhood all had a single class type. In this work, we introduce a hidden Markov model dependency (see Figure 1(d)) which assumes that pixels in neighboring locations have a high probability of being the same class. This small distinction provides two advantages: (a) we relax the problem of tuning the size of the spatial dependency region; and (b) we allow finer transitions between classes on a pixel-by-pixel basis. Future work plans to generalize the model to allow for mixtures of object classes.

\subsection{Priors on the distribution parameters}

In general, the parameters of the distributions in Table 2 are not known a priori. Instead, we propose a hierarchical Bayesian model based on the work by Tipping ${ }^{9}$ and others. ${ }^{8,10,11}$ In this model, we assume that the parameters of the distribution are also random variables that need to be estimated from the data. In most cases, we choose a non-informative distributions on the parameters in order to implement inference algorithms with few tuning parameters. Table 3 shows the distributions of the so-called 'hyper-parameters' used in this work. For this work, we assume that the covariance matrices are given by equations (6) and (9) so that they are parameterized by $\sigma^{2}, \rho$, and $\left\{\phi_{n m}\right\}_{n, m}$. Unlike the other parameters, using this structure suggests an informative prior based on our knowledge of the phenomenology of the SAR sensor. Future work will compare to the non-informative case, where $\boldsymbol{\Sigma}$ is Inverse-Wishart distributed.

As in Tipping, ${ }^{9}$ we assume that the hyperparameters on the Inverse Gamma distributions are noninformative leading to

$$
a_{\sigma}=b_{\sigma}=10^{-6}
$$

On the other hand, since the background coherence should be near unity, we let

$$
\begin{aligned}
a_{\rho} & =0.9, \\
b_{\rho} & =0.1 .
\end{aligned}
$$

It is also assumed that all object classes have equal prior probability, so that

$$
c_{n}=1 / C, \quad n=1,2, \ldots, C
$$

\subsubsection{Indicator probability models}

We have a great deal of flexibility for choosing the hyperparameters for the indicator probabilities. To ensure that only a small percentage of the pixels contain either specular noise or targets, one should set the Beta parameters so that

$$
\frac{a(p)}{a(p)+b(p)} \approx 0 .
$$

However, in many cases there is a high degree of spatial and temporal dependence for the indicator variables. For example, both targets and glints have spatial extents that spread over several pixels, while moving targets tend to transition smoothly to neighboring pixels in sequential frames. Furthermore, it may be possible to identify regions of the scene where specular noise or targets have a high likelihood, e.g. at edges of buildings 
Table 3. Distributional models for parameters of distributions in Table 2

\section{Component}

Stationary covariance

Variance

Coherence

Phases

\begin{tabular}{|l|} 
Speckle covariance \\
Variance \\
Coherence \\
Phases
\end{tabular}

Specular covariance
Variance
Coherence
Phases

Target covariance

Variance

Coherence

Phases

Additive noise covariance

Variance

Coherence

Phases

Target indicator probability

Specular indicator probability

Region type probabilities
Variable

Distribution

Parameters

$$
\boldsymbol{\Sigma}^{S}(j)
$$$$
\left(\sigma^{S}(j)\right)^{2}
$$

Inv-Gamma

$a_{\sigma}, b_{\sigma}$

$\rho^{S}(j)$

Beta

$a_{\rho}, b_{\rho}$

$\left\{\phi_{n m}^{S}(j)\right\}_{n, m}$

Deterministic

$\phi_{n m}^{S}(j)=0$

\begin{tabular}{|c|c|c|c|}
\hline $\boldsymbol{\Sigma}^{X}(j)$ & & & Each $Q_{j}$ \\
\hline$\left(\sigma^{X}(j)\right)^{2}$ & Inv-Gamma & $a_{\sigma}, b_{\sigma}$ & \\
\hline$\rho^{X}(j)$ & Beta & $a_{\rho}, b_{\rho}$ & \\
\hline$\left\{\phi_{n m}^{X}(j)\right\}_{n, m}$ & Deterministic & $\phi_{n m}^{X}(j)=0$ & \\
\hline
\end{tabular}

$$
\boldsymbol{\Sigma}^{Y}(j)
$$

Each $Q_{j}$

$\left(\sigma^{Y}(j)\right)^{2}$

Inv-Gamma

$a_{\sigma}, b_{\sigma}$

$\rho^{Y}(j)$

Beta

$a_{\rho}, b_{\rho}$

$\left\{\phi_{n m}^{Y}(j)\right\}_{n, m}$

Deterministic

$\phi_{n m}^{Y}(j)=0$

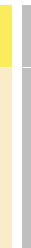$$
\Sigma^{M}
$$$$
\left(\sigma^{M}\right)^{2}
$$$$
\rho^{M}
$$$$
\left\{\phi_{n m}^{M}\right\}_{n, m}
$$$$
\text { ministic }
$$$$
\text { All } p
$$

Each $Q_{j}$

\section{Region}

Each $Q_{j}$

(

$$
\Sigma^{V}
$$$$
\left(\sigma^{V}\right)^{2}
$$$$
\rho^{V}
$$

Inv-Gamma

$a_{\sigma}, b_{\sigma}$

Deterministic

$\rho^{M}=0$

Uniform

$[0,2 \pi]$

All $p$

$\begin{array}{clc}\rho^{V} & \text { Deterministic } & \rho^{V}=0 \\ \left\{\phi_{n m}^{V}\right\}_{n, m} & \text { Uniform } & {[0,2 \pi]}\end{array}$

$\pi_{f, i}^{M}(p)$

$a_{f, i}^{M}(p), b_{f, i}^{M}(p) \quad$ Each $p, f, i$

$\pi_{f}^{Y}(p)$

Beta

$a_{f}^{Y}(p), b_{f}^{Y}(p)$

Each $p, f$

$\vec{q}=\left\{q_{1}, \ldots, q_{n}\right\} \quad$ Dirichlet $\quad\left\{c_{n}\right\}_{n=1}^{C} \quad$ All $p$


in the former case and near road intersections in the latter case. This information may be learned adaptively through observed data or through human-in-the loop processing. In any of these cases, one would expect that

$$
\frac{a(p)}{a(p)+b(p)} \approx 1
$$

Define $W_{f}^{Y}(p, \boldsymbol{\delta})$ and $W_{f, i}^{M}(p, \boldsymbol{D})$ to be functions that map the indicator variables $\boldsymbol{\delta}$ and $\boldsymbol{D}$, respectively, to a real number. For example, this may be the average number of non-zero indicators in the neighborhood of pixel $p$, or it may be a weighted version similar to Figure $1(\mathrm{~d})$. Then define:

$$
a_{f}^{Y}(p)=\left\{\begin{array}{ll}
a_{\text {high }}, & W_{f}^{Y}\left(p, \boldsymbol{\delta}_{f}\right)>\varepsilon_{\text {spatial }}^{Y} \\
a_{\text {low }}, & \text { else }
\end{array}, \quad b_{f}^{Y}(p)= \begin{cases}b_{\text {high }}, & W_{f}^{Y}\left(p, \boldsymbol{\delta}_{f}\right)>\varepsilon_{\text {spatial }}^{Y} \\
b_{\text {low }}, & \text { else }\end{cases}\right.
$$

and

$$
\begin{aligned}
& a_{f, i}^{M}(p)= \begin{cases}a_{\text {high }}, & W_{f, i}^{M}\left(p, \boldsymbol{D}_{f, i}\right)>\varepsilon_{\text {spatial }}^{M} \text { and } W_{f, i}^{M}\left(p, \boldsymbol{D}_{f-1, i}\right)>\varepsilon_{\text {temporal }}^{M} \\
a_{\text {low }}, & \text { else }\end{cases} \\
& b_{f, i}^{M}(p)= \begin{cases}b_{\text {high }}, & W_{f, i}^{M}\left(p, \boldsymbol{D}_{f, i}\right)>\varepsilon_{\text {spatial }}^{M} \text { and } W_{f, i}^{M}\left(p, \boldsymbol{D}_{f-1, i}\right)>\varepsilon_{\text {temporal }}^{M} \\
b_{\text {low }}, & \text { else. }\end{cases}
\end{aligned}
$$

Note that $\left(a_{\text {low }}, b_{\text {low }}\right)$ should be chosen to satisfy equation $(17)$ and $\left(a_{\text {high }}, b_{\text {high }}\right)$ should be chosen to satisfy equation (18).

\subsection{Generalizability of the model}

The authors would like to stress that the model presented here is easily generalized to include additional information sources. For example, if a target's state (e.g. position and velocity), then one could predict the location of its signature within the scene with high probability (see $\mathrm{Jao}^{2}$ or Newstadt et. al. ${ }^{13}$ ). Moreover, one might consider different target classes or explicitly estimating the target phase which is proportional to its radial velocity.

\section{POSTERIOR INFERENCE}

In this work, the computation of the posterior distribution estimation is done using a Gibb sampling scheme, where the vast majority of variables can be easily sampled from simple distributions such as the multivariatenormal and beta distributions. Since we wish to use the structure of the antenna covariance given in equation (9), sampling is more difficult and requires other sampling methods, such as the Metropolis-Hastings algorithm. Appendix A provides pseudocode for the implementation of our sampling method.

\section{PERFORMANCE ANALYSIS}

Figure 2 shows the full scene used for our performance analysis. The images are formed from a superset of the data available through the public-released SAR-GMTI challenge problem. ${ }^{14}$ Images were formed for a 3 second interval from three passes of the radar platform and for each of three antennas. We considered five separate locations that contained the signature of the Durango truck from the SAR-GMTI challenge problem at times $t_{f}, f=1,2, \ldots, 5$. Figure 3 shows images of the scenes created at each of the times $t_{f}$. These images contain a variety of scenarios, including stationary targets, weak target signatures, strong target signatures $\{(4,4)\}$ and barely visible target signatures. 


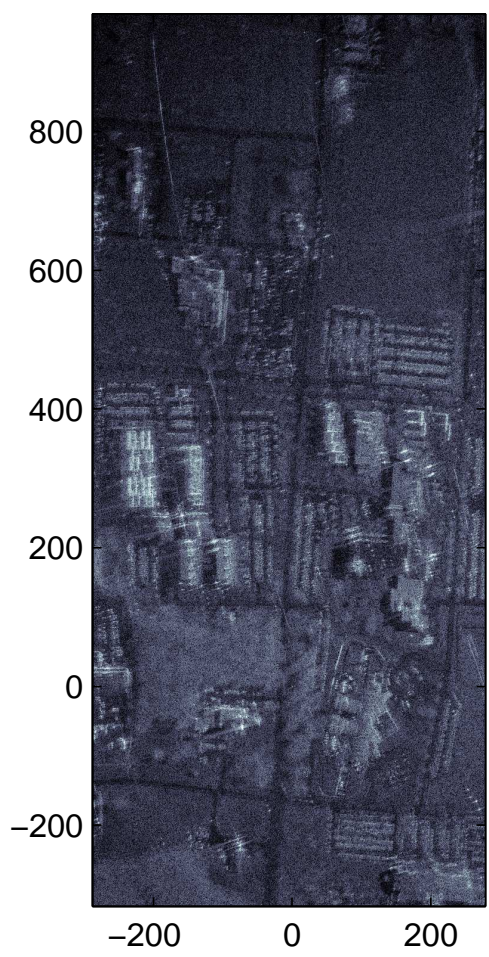

(a) Full scene

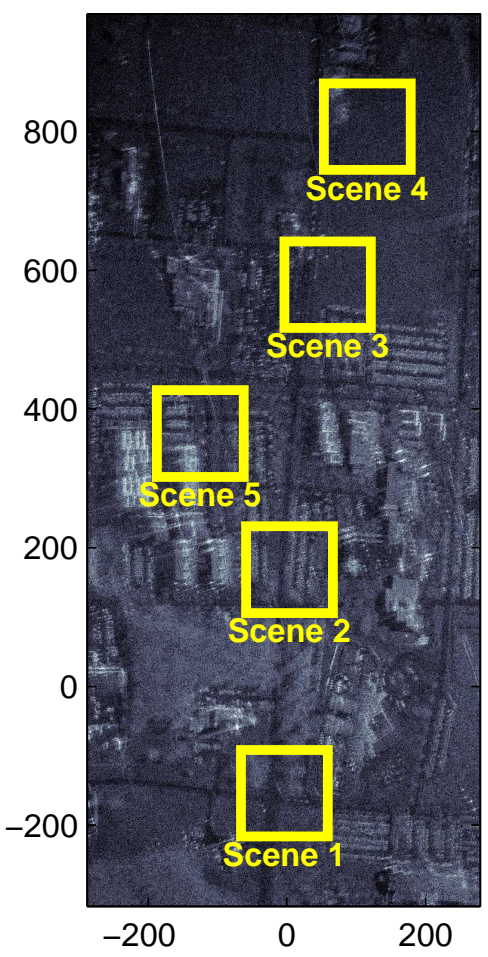

(b) Monitored Regions

Figure 2. The full scene used for the performance analysis is shown in (a), while the yellow boxes in (b) correspond to the individual scenes used for comparison of various scenarios. Note that the images formed in this dataset are a superset of the public-released SAR-GMTI challenge problem dataset. ${ }^{14}$

Figure 4 shows the output of our algorithm for the scene/time combinations, $\{(1,1),(2,2),(3,3),(4,4),(5,5)\}$ (i.e., the scenes containing the Durango truck signature), as well as comparisons to DPCA and a mixture of DPCA and ATI. For clarity, a grayscale image refers to a deterministic output such as the original image or DPCA output, while a colored image refers to a probability or variance parameter. It can be seen that our algorithm performs at least as well both DPCA and the DPCA/ATI mixture, although usually with fewer false alarms. Moreover, both of the alternative algorithms required tuning of the threshold parameters (for each of the 5 scenes). Finally, our method provides estimates of the posterior distribution, such as the variance of the background (which could be used for stronger hypothesis testing procedures) and the probability of target existence.

Figure 5 compares the performance of our algorithm for scenes 2 and 3 as a function of the number of observations. In particular, images were formed for each scene from $n$ distinct look-angles, where $n \in$ $\{10,20,30,40,50\}$. Each 'observation' consists of a set of images formed for $K=3$ antennas and $N=3$ passes. It can be seen that as the number of observations grows, the performance of our algorithm improves with fewer false alarms.

In Figure 6, we compare the performance for scenes 2 and 3 as a function of the integration length $(\{0.2,0.5,1.0\}$ seconds $)$ used to form the images. It should be noted that for each integration length, a set of images was formed that encompassed the entire 3 second interval (although possibly with fewer/greater number of frames, $F$ ). Thus, this simulation compares the robustness of our algorithm to using an arbitrary integration length. The results suggest that the performance of our algorithm is invariant to the choice of integration length. This relaxes the selection of another tuning parameter that can affect performance in other algorithms such as DPCA and ATI. 


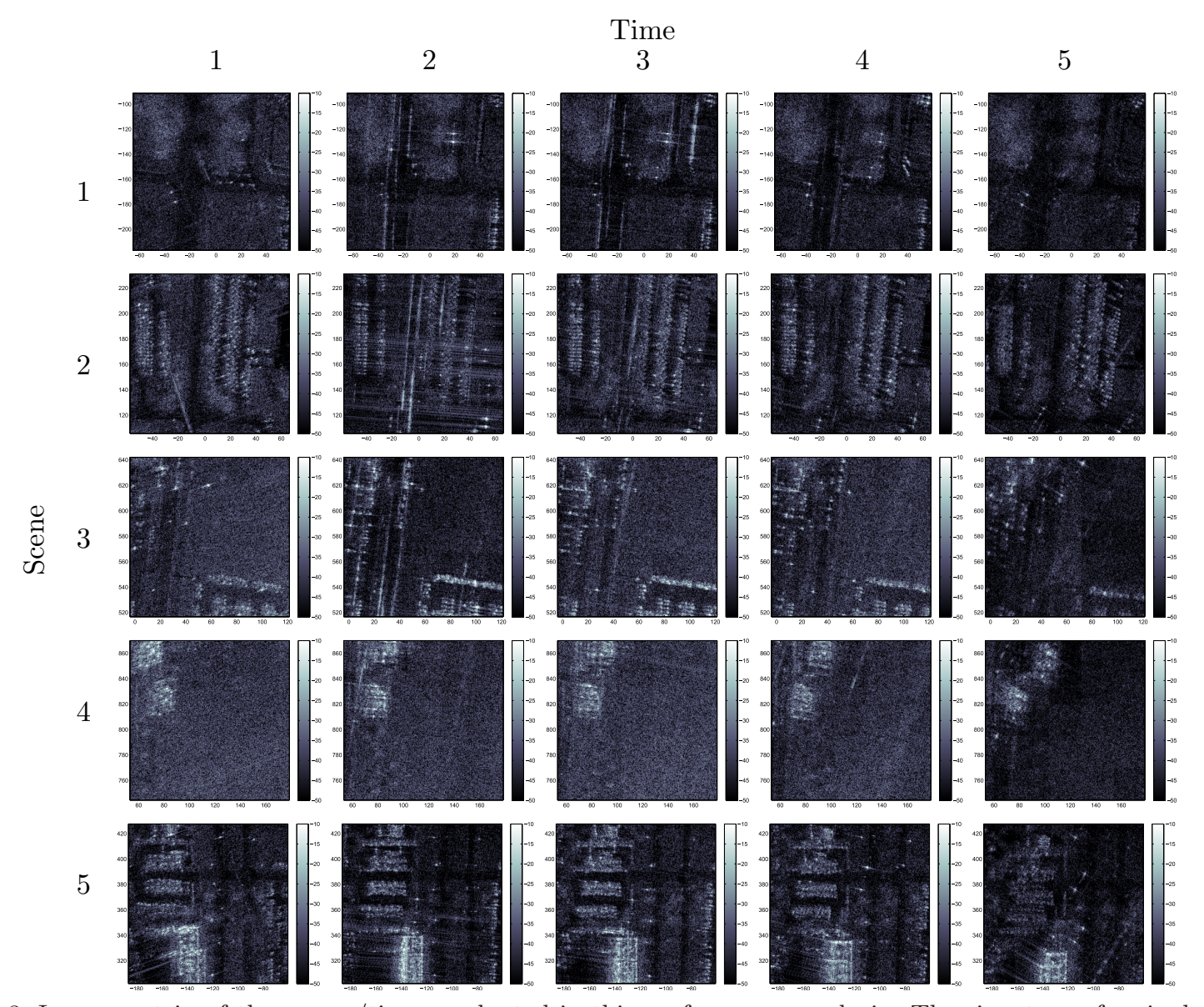

Figure 3. Image matrix of the scenes/times evaluated in this performance analysis. The signature of a single target (Durango Truck) is known to exist along the diagonal, although other targets are in other scenes. Rows of the matrix correspond to the same location as in Figure 2(b), while columns correspond to different times (i.e., azimuth ranges). It should be noted that there are stationary targets $\{(1,1)\}$, weak target signatures $\{(2,2),(3,3)\}$, strong target signatures $\{(4,4)\}$ and barely visible target signatures $\{(5,5)\}$. 
Original $\quad E\left[B_{k, f, i}(p)\right] \operatorname{Var}\left[B_{k, f, i}(p)\right] E\left[T_{k, f, i}(p)\right] \operatorname{Pr}\left(D_{f, i}(p)\right) \quad$ DPCA $\quad$ DPCA/ATI

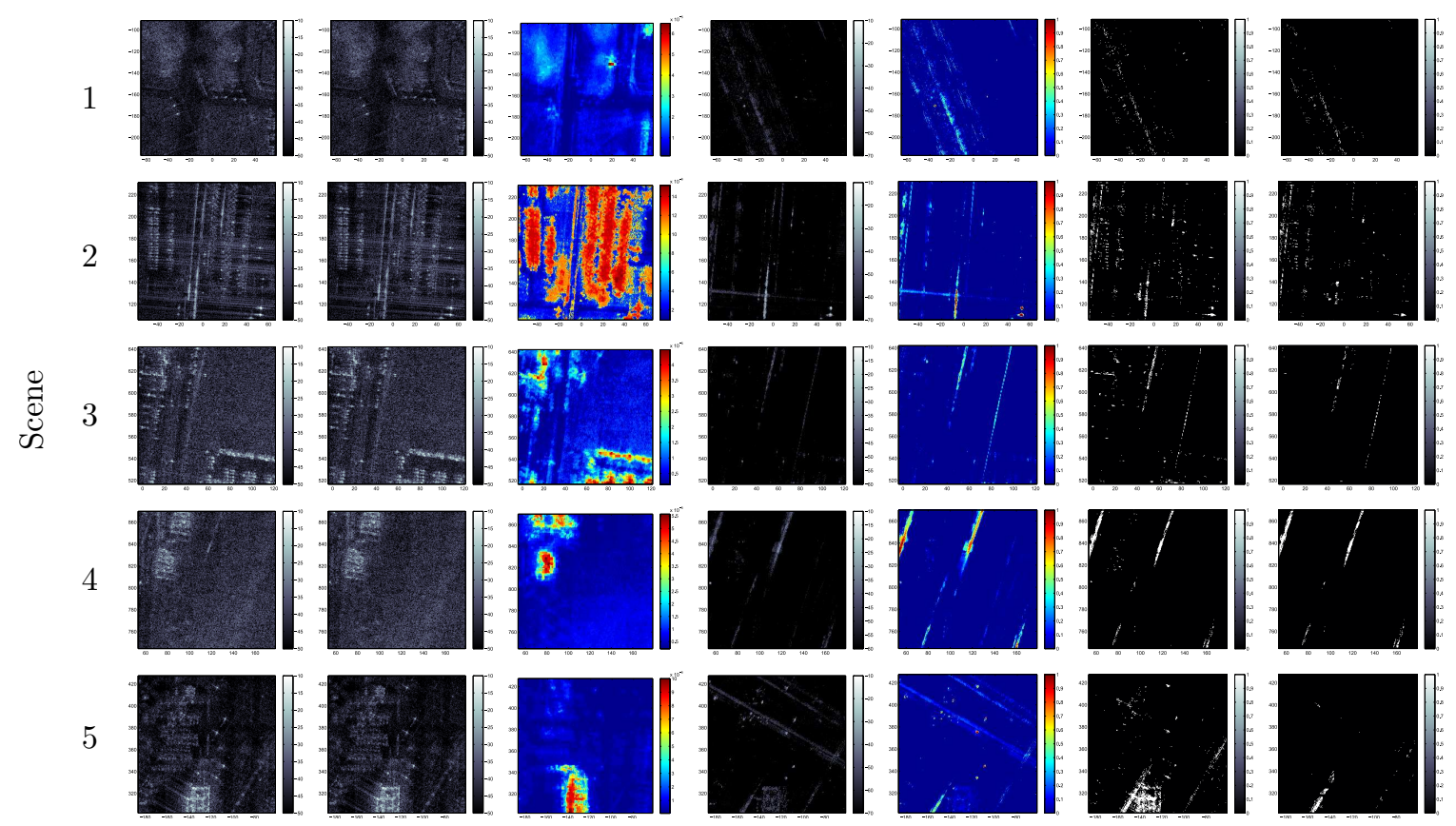

Figure 4. This figure shows the output of our algorithm for the scene/time combinations for the scenes containing the Durango truck signature, $\{(1,1),(2,2),(3,3),(4,4),(5,5)\}$. Grayscale images refer a deterministic output such as the original image or DPCA output, while colored images refer to a probability or variance parameter. It is seen that our algorithm performs at least as well both DPCA and the DPCA/ATI mixture, although usually with fewer false alarms.

Number of Observations

\section{Original}

10

20

30

40

50

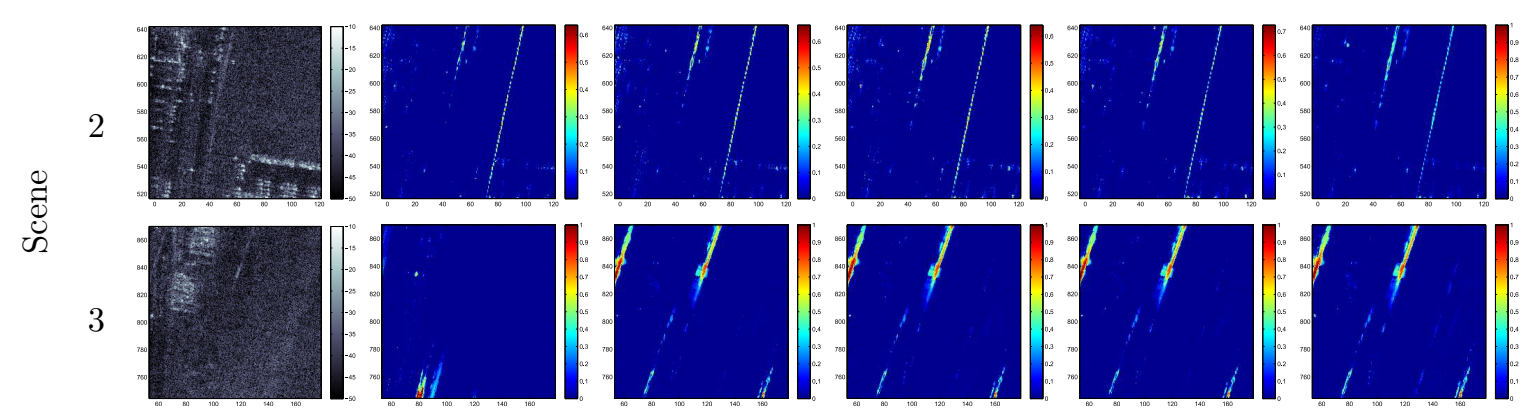

Figure 5. This figure compares the performance of our proposed method for scenes 2 and 3 as a function of the number of observations, $n$. A single observation consists of a set of images formed at a set of azimuth (look-) angles $\theta_{n}$ for each of $K=3$ antennas and $N=3$ passes. The number of observations was varied for $n \in\{10,20,30,40,50\}$. It can be seen that as $n$ grows, the performance of our algorithm improves with fewer false alarms. Moreover, the performance of the algorithm is relatively consistent for larger $n$, which suggests that the estimation of the background distribution can be done with relatively few observations. 


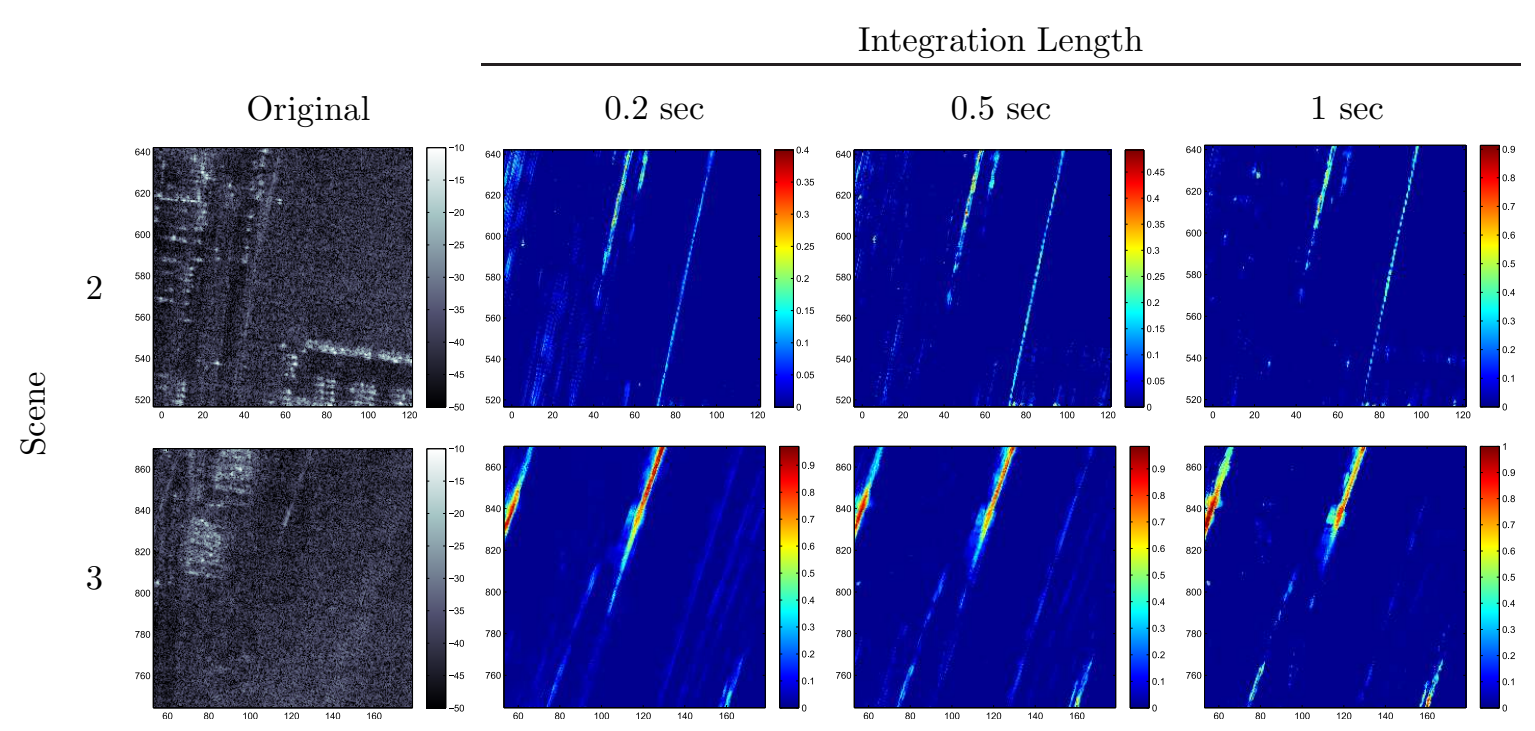

Figure 6. This figure compares the performance of our proposed method for scenes 2 and 3 as a function of the integration length $(\{0.2,0.5,1.0\}$ seconds) used to form the images. The results suggest that the performance of our algorithm is invariant to the choice of integration length, which relaxes the selection of another tuning parameter that can affect performance in other algorithms such as DPCA and ATI.

Figure 7 shows the output of our algorithm both with and without the new antenna covariance model. It can be seen that using the covariance model significantly reduces the number of false alarms in areas where the background has high variance.

Figure 8 shows the output of our algorithm when prior information on the location of the targets might be available. For example, in scene 1, targets are likely to be stopped at an intersection. We show the performance improvement for two scenes that contain targets in our high probability region (mission) and that don't contain targets in those regions (reference). It can be seen that by including the prior information, we are able to detect targets with much better accuracy, including the stationary targets in scene 1 . On the other hand, we don't have significant performance decreases in the reference scenarios.

\section{CONCLUSIONS AND FUTURE WORK}

In this paper we extended the development and analysis of a hierarchical Bayesian model for persistent SAR imagery, along with a Gibbs sampling scheme to efficiently estimate the posterior distribution. This algorithm can infer statistics of the noise without extensive tuning of hyperparameters, yet also provides a characterization of its uncertainty through a posterior distribution. The previous model was extended by estimating the antenna covariance matrix directly, leading to improved performance in high clutter regions. The model is also shown to be easily generalizable when additional prior information is available, such as locations of roads/intersections or smoothness priors on the target motion. The performance of our posterior inference algorithm is analyzed over a large set of measured SAR imagery. It is shown that the proposed algorithm works at least as well as common change detection algorithms. Moreover, the algorithm is shown to work well in a number of cases that include detecting stationary targets at intersections, detecting targets 


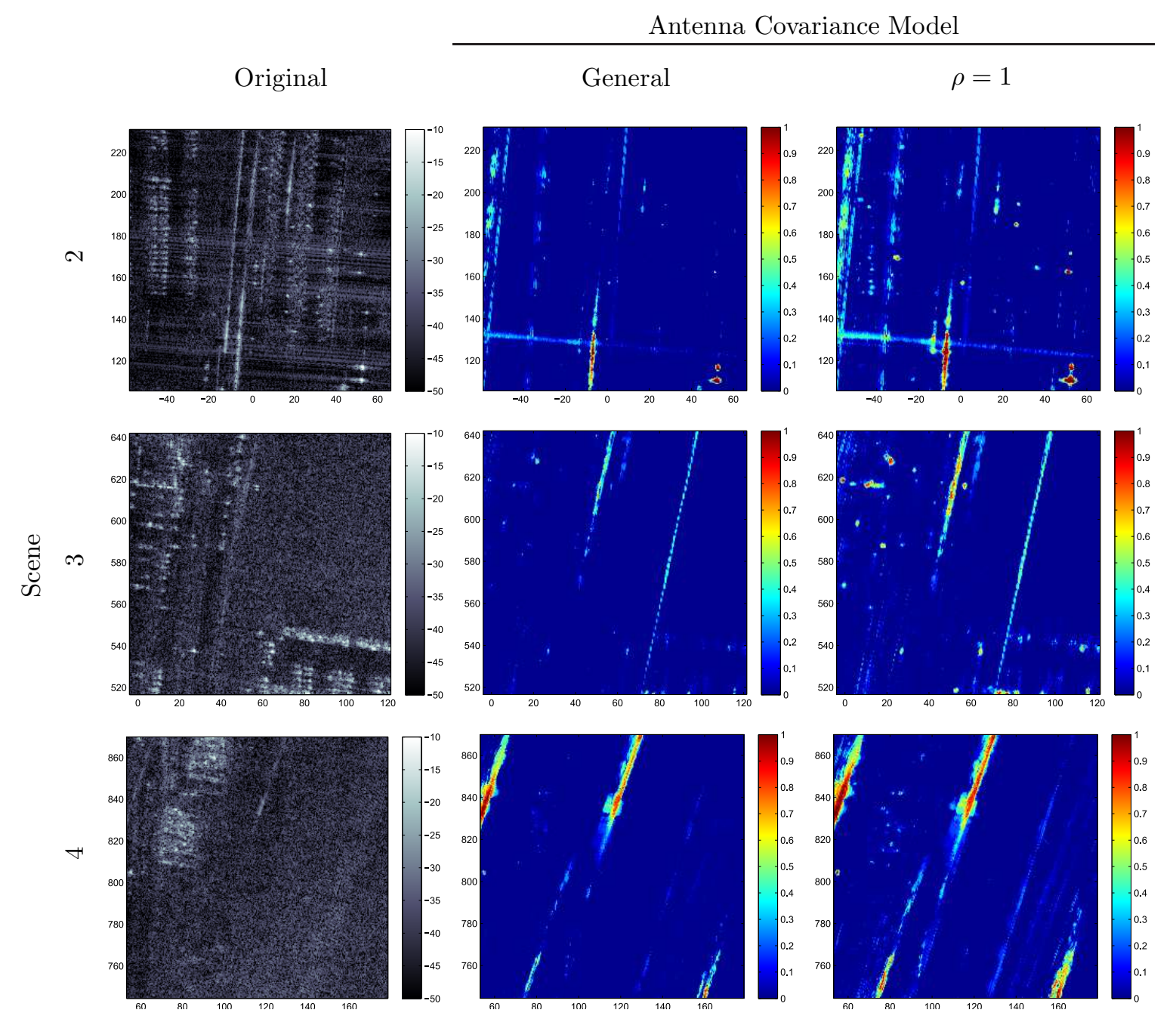

Figure 7. This figure compares the performance of our proposed method both with the new antenna covariance model and as well the simpler model where the coherence of the channels is set to $\rho=1$. It can be seen that using the more general covariance model significantly reduces the number of false alarms in areas where the background has high variance (i.e, high clutter regions.) 
Target Prior Model

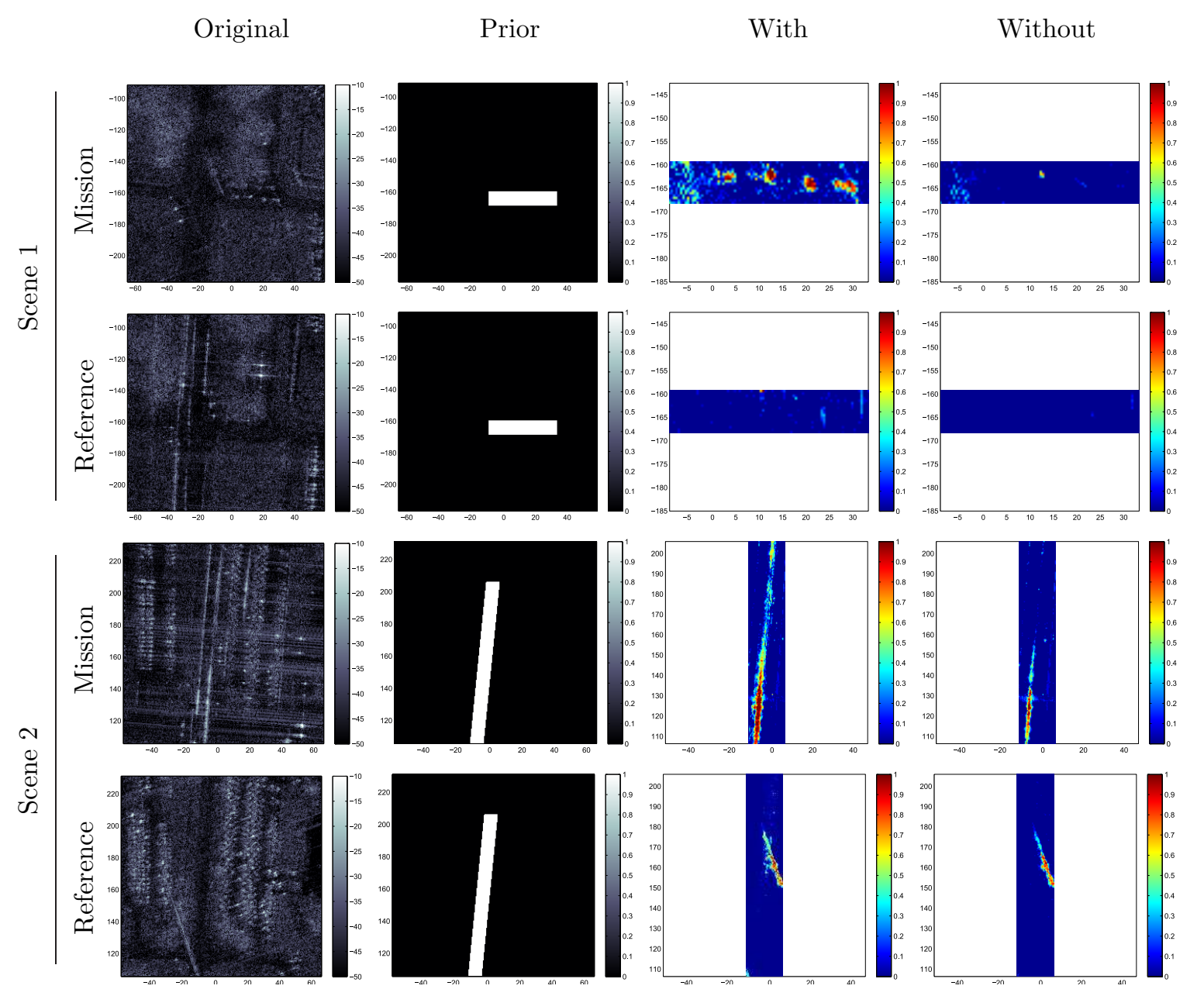

Figure 8. This figure compares the performance of our proposed method with and without priors on target signature locations. In scene 1, targets are likely to be stopped at an intersection, while in scene 2 , the target signature is assumed to lie in a low clutter region. We show the performance improvement for two scenes that contain targets in the high probability region (mission scenes) and for two scenes that don't contain targets in those regions (reference scenes). It can be seen that by including the prior information, we are able to detect targets with much better accuracy, including the stationary targets in scene 1. On the other hand, we don't have significant performance decreases in the reference scenarios. 
in both bright and dim clutter situations, and working well regardless of the integration length used to form the SAR images.

Future work will include the development of algorithms that exploit the use of a posterior distribution for improved performance in a signal processing task, e.g. detection, tracking or classification. In particular, we are interested in using algorithms for simultaneously detecting and estimating targets over a sparse scene with resource constraints ${ }^{15,16}$ as well determining the fundamental performance limits of a SAR target tracking system. Furthermore, we would also like to consider other generalizations to our model, such as complex target maneuvers, multiple target classes, and explicit tracking of the target phase.

\section{Acknowledgment}

This research was partially supported by the Air Force Research Laboratory Sensors Directorate under grant FA8650-07-D-1220-0006.

\section{REFERENCES}

[1] G. Newstadt, E. Zelnio, and A. Hero III, "Persistent SAR Change Detection with Posterior Models," Proc. of SPIE Vol, vol. 8051, pp. 80510R-1, 2011.

[2] J. Jao, "Theory of synthetic aperture radar imaging of a moving target," IEEE Transactions on Geoscience and Remote Sensing, vol. 39, no. 9, pp. 1984-1992, 2001.

[3] J. Fienup, "Detecting moving targets in SAR imagery by focusing," IEEE Transactions on Aerospace and Electronic Systems, vol. 37, no. 3, pp. 794-809, 2001.

[4] B. Borden and M. Mumford, "A statistical glint/radar cross section target model," Aerospace and Electronic Systems, IEEE Transactions on, vol. AES-19, no. 5, pp. 781 -785, 1983.

[5] M. Soumekh, "Moving target detection in foliage using along track monopulse synthetic aperture radar imaging," Image Processing, IEEE Transactions on, vol. 6, no. 8, pp. 1148 -1163, Aug. 1997.

[6] K. Ranney and M. Soumekh, "Signal subspace change detection in averaged multilook sar imagery," Geoscience and Remote Sensing, IEEE Transactions on, vol. 44, no. 1, pp. 201 - 213, jan. 2006.

[7] J. Ender, "Space-time processing for multichannel synthetic aperture radar," Electronics and Communication Engineering Journal, vol. 11, no. 1, pp. 29-38, 1999.

[8] X. Ding, L. He, and L. Carin, "Bayesian Robust Principal Component Analysis," submitted for publication, 2010.

[9] M. Tipping, "Sparse Bayesian learning and the relevance vector machine," The Journal of Machine Learning Research, vol. 1, pp. 211-244, 2001.

[10] D. P. Wipf and B. D. Rao, "Sparse Bayesian learning for basis selection," IEEE Transactions on Signal Processing, vol. 52, no. 8, pp. 2153-2164, August 2004.

[11] D. Wipf, J. Palmer, and B. Rao, "Perspectives on sparse Bayesian learning," in Advances in neural information processing systems 16: proceedings of the 2003 conference, vol. 16. The MIT Press, 2004, p. 249.

[12] R. Deming, "Along-track interferometry for simultaneous sar and gmti: application to gotcha challenge data," in Proceedings of SPIE, vol. 8051, 2011, p. 80510P.

[13] G. E. Newstadt, E. Zelnio, L. Gorham, and A. O. H. III, "Detection/tracking of moving targets with synthetic aperture radars," E. G. Zelnio and F. D. Garber, Eds., vol. 7699, no. 1. SPIE, 2010, p. 76990I. [Online]. Available: http://link.aip.org/link/?PSI/7699/76990I/1

[14] S. M. Scarborough, C. H. Casteel Jr, L. Gorham, M. J. Minardi, U. K. Majumder, M. G. Judge, E. Zelnio, M. Bryant, H. Nichols, and D. Page, "A challenge problem for SAR-based GMTI in urban environments," E. G. Zelnio and F. D. Garber, Eds., vol. 7337, no. 1. SPIE, 2009, p. 73370G. [Online]. Available: http://link.aip.org/link/?PSI/7337/73370G/1 


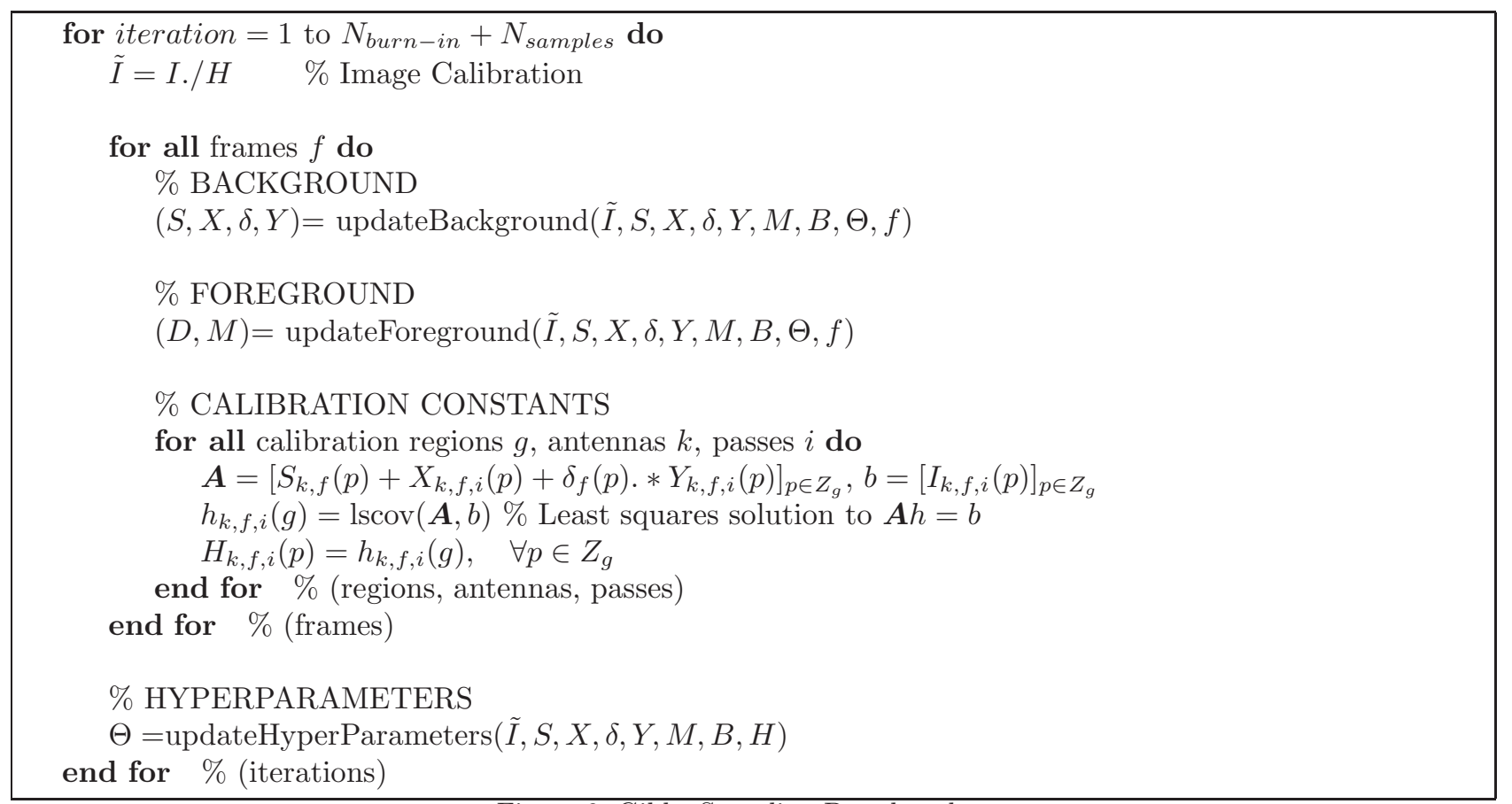

Figure 9. Gibbs Sampling Pseudocode

[15] E. Bashan, R. Raich, and A. O. H. III, "Optimal two-stage search for sparse targets using convex criteria," IEEE Transaction Signal Processing, vol. 56, no. 11, pp. 5389-5402, November 2008.

[16] E. Bashan, G. Newstadt, and A. Hero, "Two-Stage Multi-Scale Search for Sparse Targets," IEEE Transactions on Signal Processing, vol. 59, no. 5, pp. 2331-2341, 2011.

\section{APPENDIX A. PSEUDOCODE FOR POSTERIOR ESTIMATION}

Figures 9 through 16 provide the pseudocode used for our posterior estimation algorithm. 


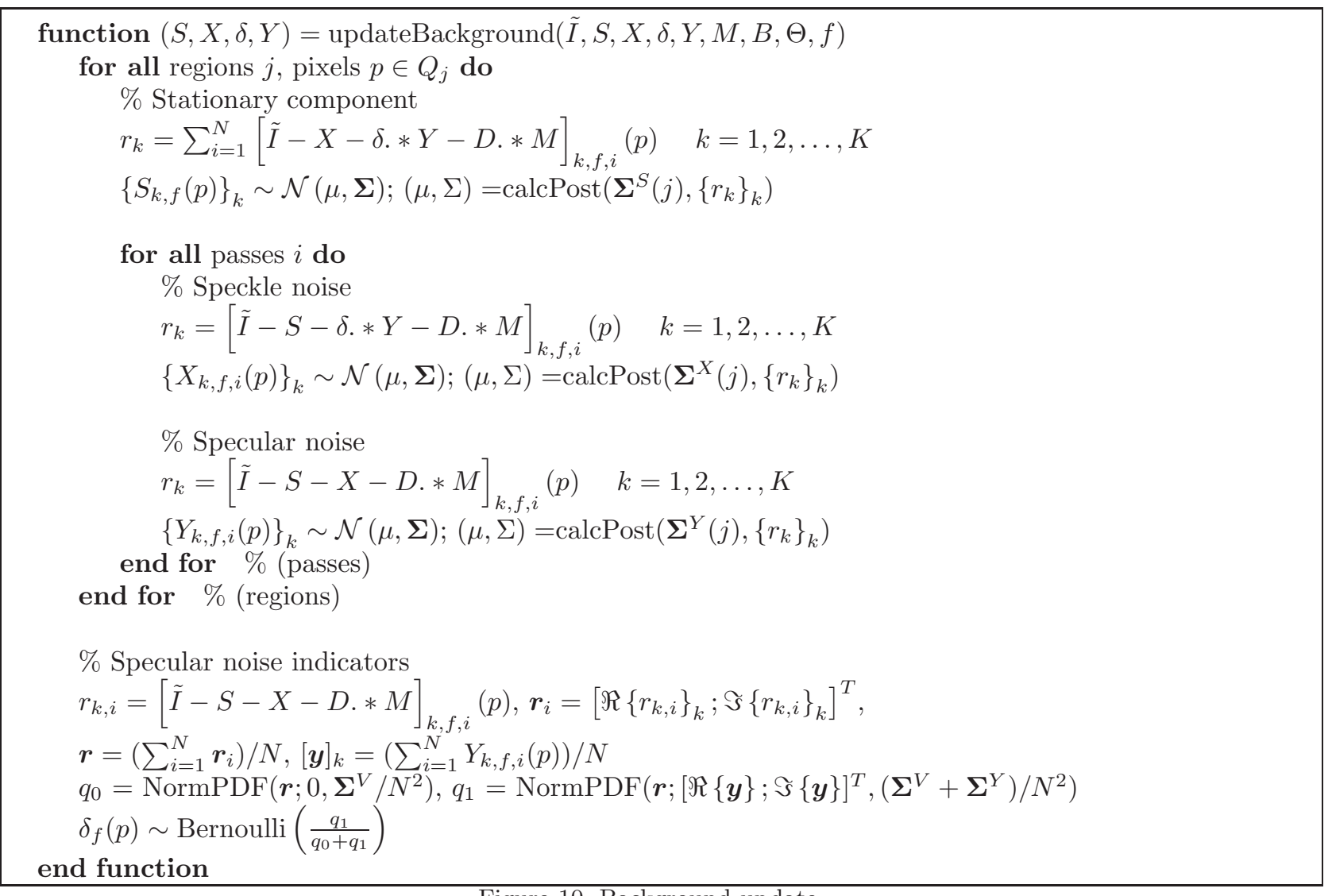

Figure 10. Background update

function $(D, M)=$ updateForeground $(\tilde{I}, S, X, \delta, Y, M, B, \Theta, f)$

for all passes $i$ do

$\%$ Target amplitudes

$r_{k}=[\tilde{I}-S-X-\delta . * Y]_{k, f, i}(p) \quad k=1,2, \ldots, K$
$\left\{M_{k, f, i}(p)\right\}_{k} \sim \mathcal{N}(\mu, \boldsymbol{\Sigma}) ;(\mu, \Sigma)=\operatorname{calcPost}\left(\boldsymbol{\Sigma}^{M},\left\{r_{k}\right\}_{k}\right)$

$\%$ Specular noise indicators

$\boldsymbol{r}=\left[\Re\left\{r_{k}\right\}_{k} ; \Im\left\{r_{k}\right\}_{k}\right]^{T},[\boldsymbol{m}]_{k}=M_{k, f, i}(p)$

$q_{0}=\operatorname{NormPDF}\left(\boldsymbol{r} ; 0, \boldsymbol{\Sigma}^{V}\right)$

$q_{1}=\operatorname{NormPDF}\left(\boldsymbol{r} ;[\Re\{\boldsymbol{y}\} ; \Im\{\boldsymbol{y}\}]^{T}, \boldsymbol{\Sigma}^{V}+\boldsymbol{\Sigma}^{M}\right)$

$D_{f, i}(p) \sim \operatorname{Bernoulli}\left(\frac{q_{1}}{q_{0}+q_{1}}\right)$

end for $\%$ (passes)

end function 


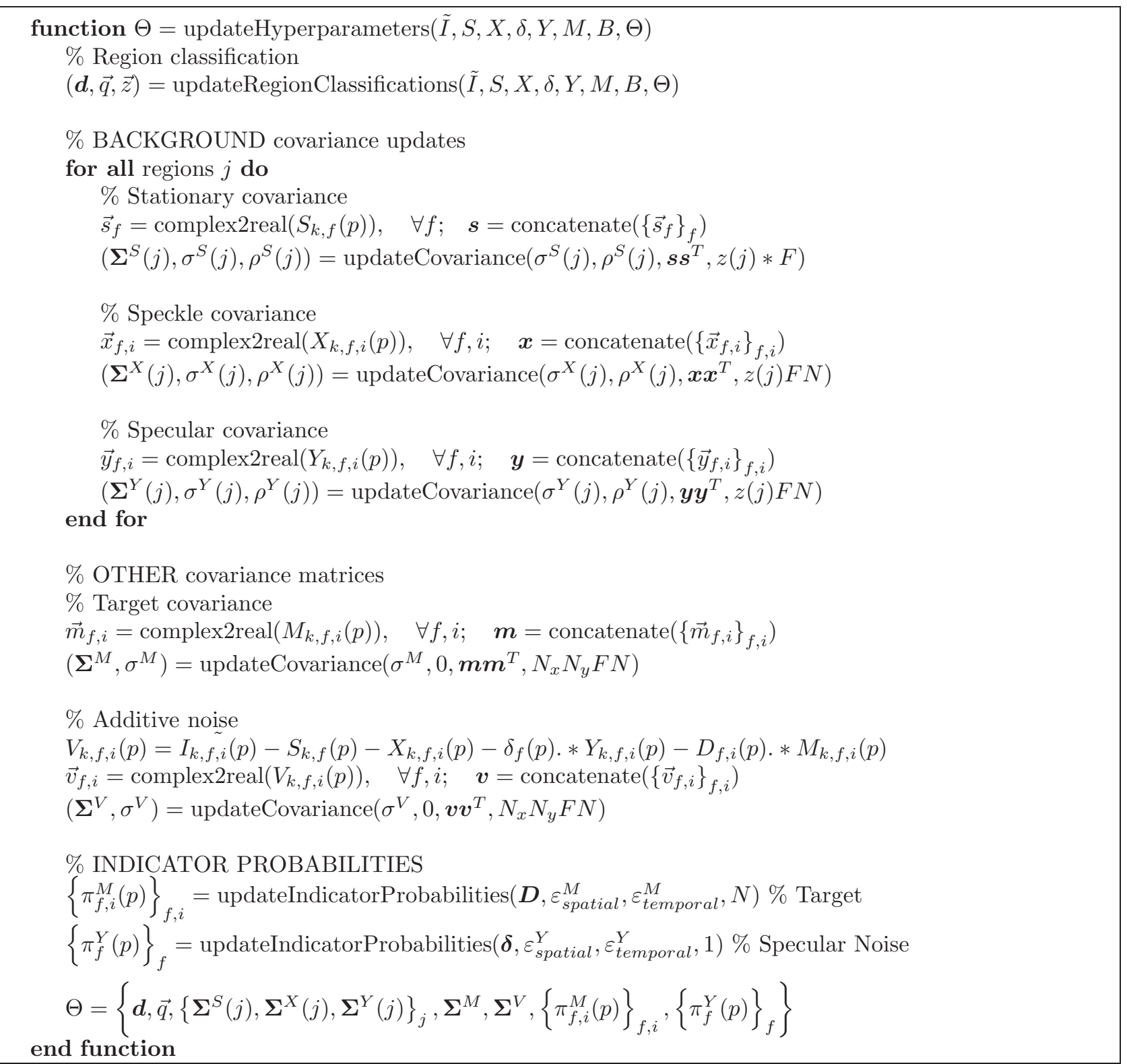




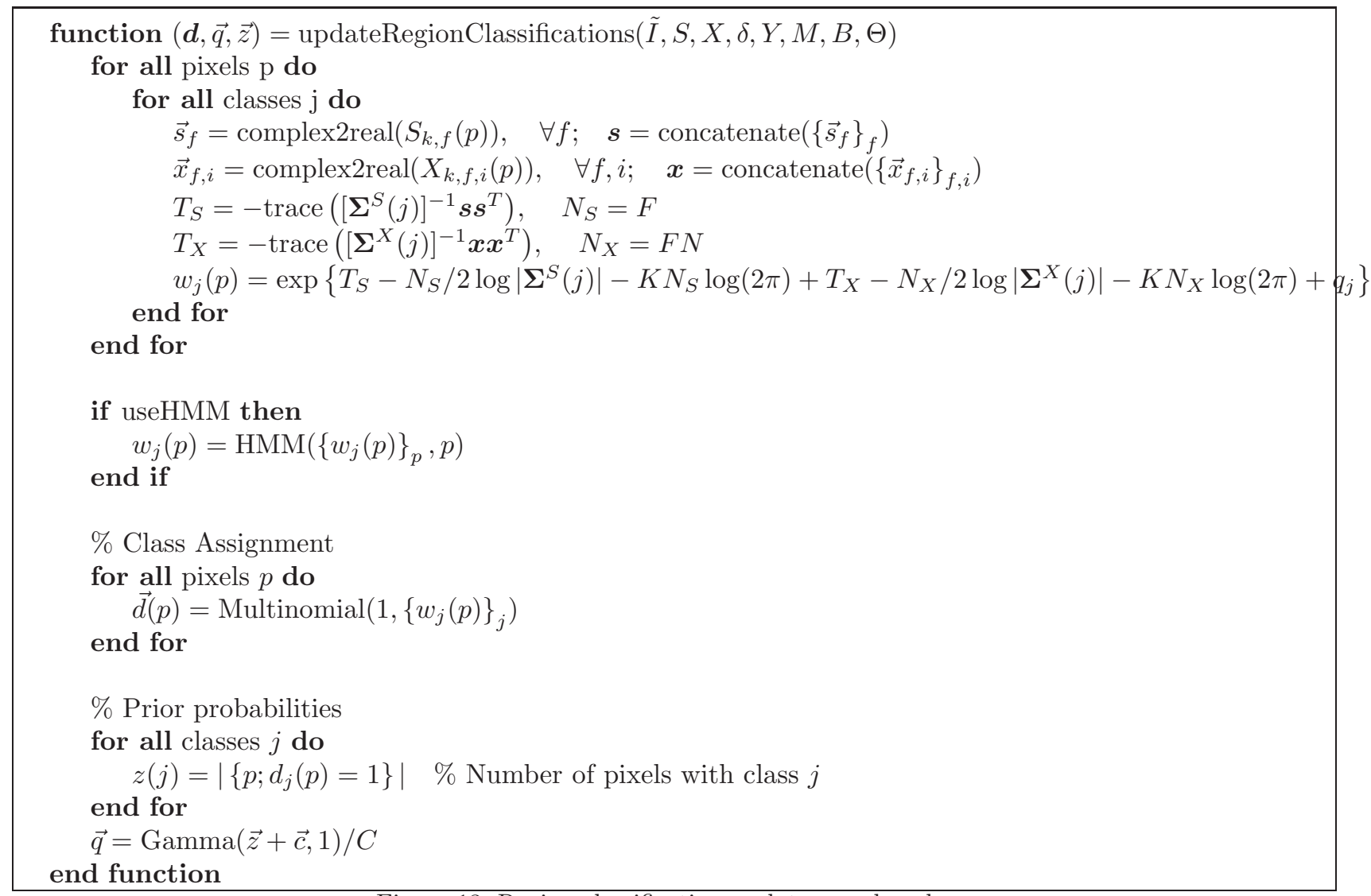

Figure 13. Region classification update pseudocode 


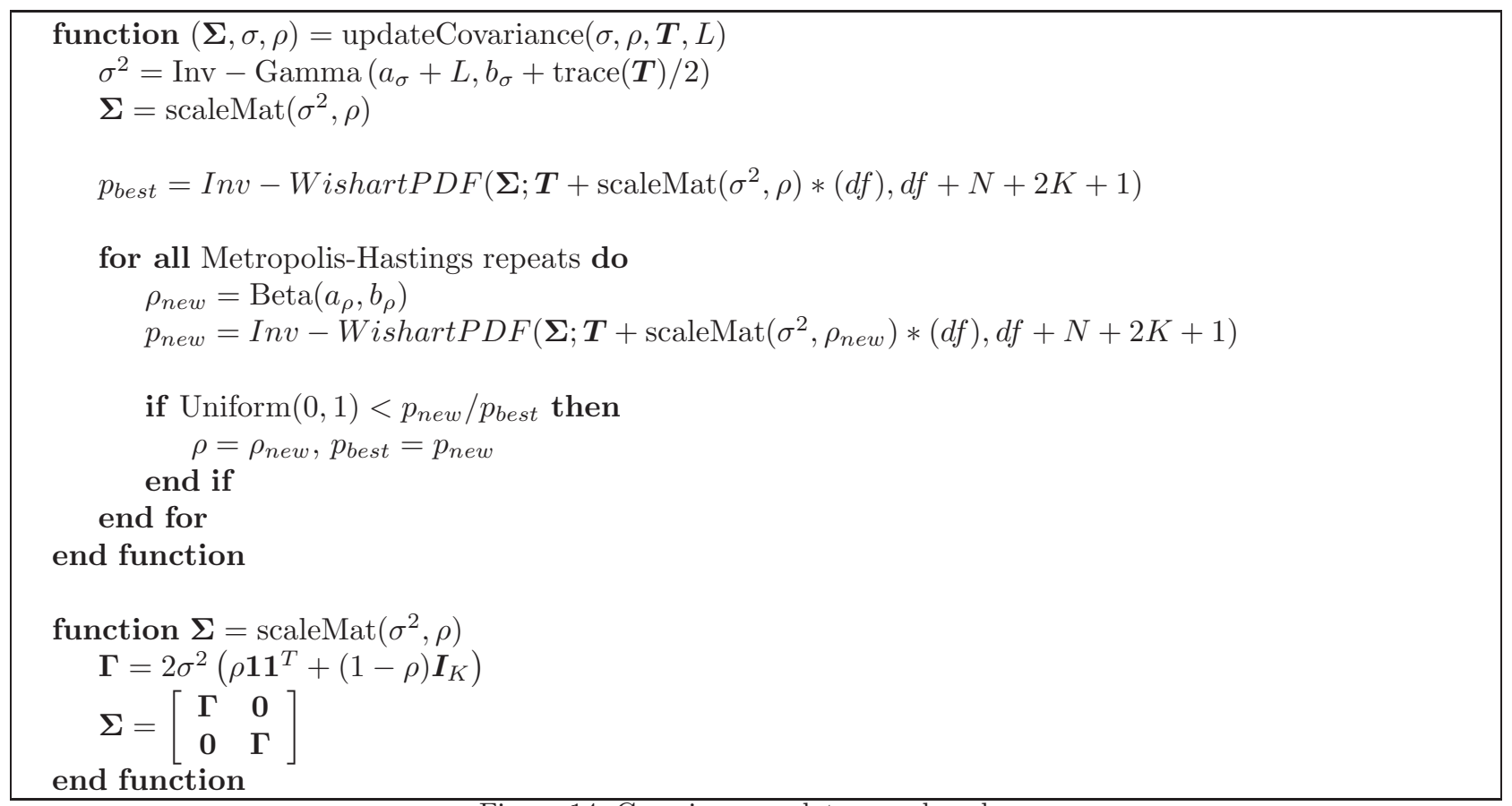

Figure 14. Covariance update pseudocode

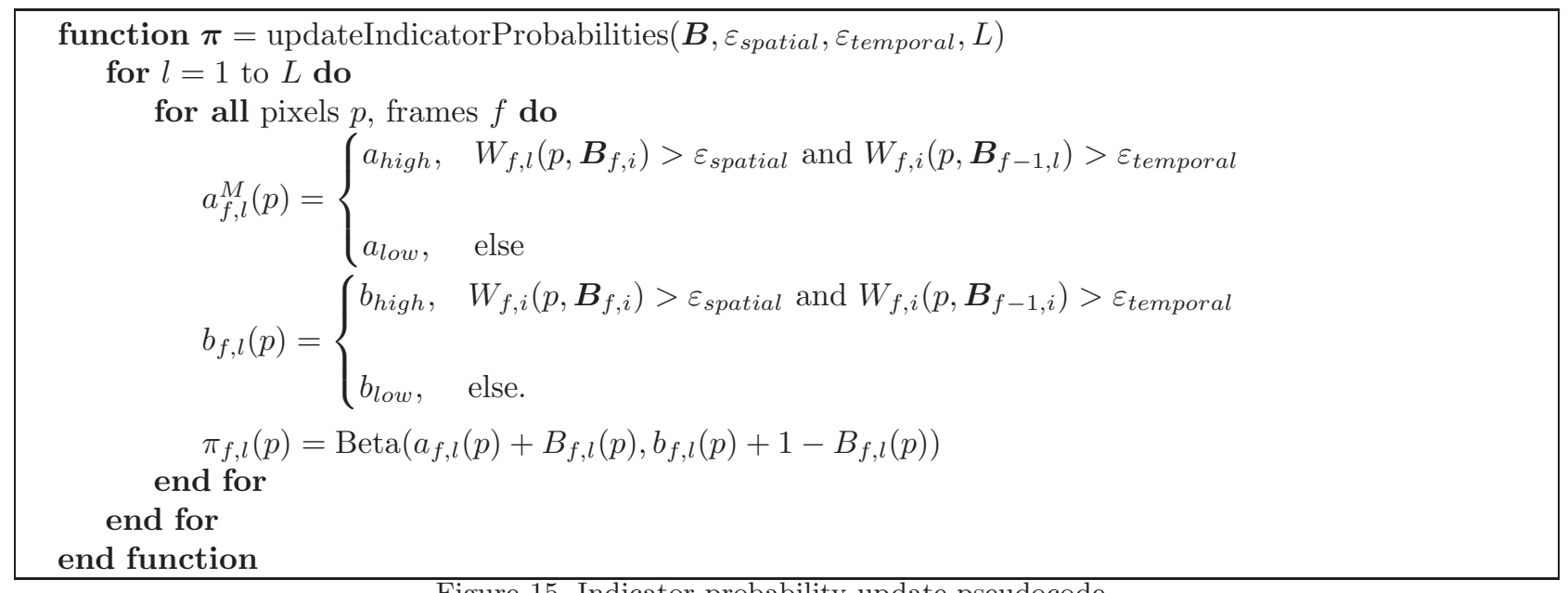




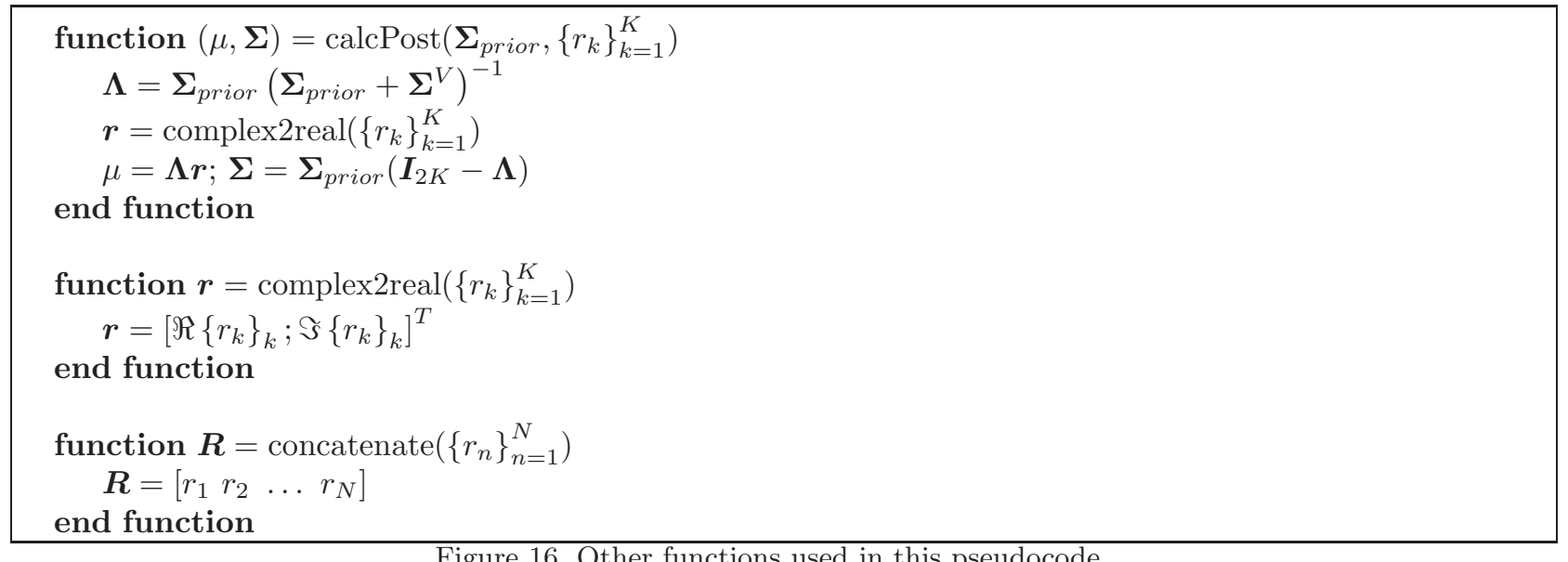

Figure 16. Other functions used in this pseudocode 